\title{
Pumping Tests in Nonuniform Aquifers: The Radially Asymmetric Case
}

\author{
JAMES J. BUTLER, JR., AND WENZHI LiU
}

Kansas Geological Survey, University of Kansas, Lawrence

\begin{abstract}
An analytical solution for the case of transient, pumping-induced drawdown in a nonuniform aquifer is presented. The nonuniform aquifer is conceptualized as a uniform matrix into which a disk of anomalous properties has been placed. The disk can be arbitrarily located with respect to the pumping well. This solution can be used to develop considerable insight concerning the nature of drawdown in nonuniform systems. Changes in drawdown are sensitive to the hydraulic properties of a discrete portion of an aquifer for a time of limited duration. After that time, it is virtually impossible to gain further information about those properties. The volume of the aquifer controlling a given increment of drawdown at an observation well increases greatly as the distance between the pumping and observation well increases. At observation wells located at moderate to large distances from the pumping well, this volume is so large that the effect of spatial variations in flow properties may be negligible. In general, drawdown data from wells located at a distance from the pumping well should nicely fit the ideal models of the well hydraulics literature. When combined with previous work, these results demonstrate that constant rate pumping tests are not an effective tool for characterizing lateral variations in flow properties.
\end{abstract}

\section{INTRODUCTION}

Analytical solutions for drawdown in response to pumping at a central well are the basis of conventional pumping-test analysis methodology. For the most part, these solutions consider hydraulic behavior in an idealized aquifer in which media properties are invariant in space. Natural systems, however, may be characterized by a considerable degree of property variability. The issue of the validity of conventional pumping-test analysis methodology in highly variable systems has not yet been resolved. This article attempts to address this issue by presenting an analytical solution for flow to a well in a laterally nonuniform configuration that the authors believe has not previously been fully considered. Evaluation of this solution provides valuable insight into the viability of conventional pumping-test methodology in many types of nonuniform aquifers.

A number of workers have presented analytical solutions for flow to a well in a laterally nonuniform aquifer. For the most part, work has concentrated on units composed of two components separated by a linear or radial discontinuity. Streltsova [1988] and Butler and Liu [1991] summarize the majority of this past work. Few investigators have addressed the case of transient flow in a system in which the aquifer nonuniformity consists of a pod of material arbitrarily located in a matrix of differing properties. Jaeger [1944] outlines a solution for the flow of heat in response to an instantaneous line source located anywhere within a circular disk whose properties differ from the surrounding matrix. Larkin [1963] briefly extends the solution to the case of a constant discharging well within the circular pod. Neither author, however, discusses the insight that might be derived from the solution. Grader and Horne [1988] consider the influence of a circular pod on drawdown when both the pumping and observation wells are located outside of the pod. Their approach, however, requires that the pod be

Copyright 1993 by the American Geophysical Union.

Paper number 92WR02128.

0043-1397/93/92WR-02128\$05.00 either impermeable or at a constant head. Wheatcraft and Winterberg [1985] investigate radial and uniform steady state flow in an aquifer consisting of a circular disk of finite permeability embedded in a matrix of differing properties.

In this article, the solution of Jaeger [1944] is extended to the general case of pumping and observation wells located anywhere within the flow system. Although this is apparently the first general presentation of the extended solution of Jaeger, the details of the analytical solution are relegated to an appendix. The major purpose of this paper is to demonstrate how this solution can be used to develop insight into pumping-induced flow in nonuniform aquifers. Two techniques will be employed as part of this effort: sensitivity analysis [McElwee, 1987] and large-time approximations of the analytical solution. Unlike most contributions in the well-hydraulics literature, the emphasis of this work is not on the development of a new analytical technique for application to a particular flow configuration. Instead, the emphasis is on the development of insight into how aquifer nonuniformities affect pumping-induced drawdown, so that field practitioners will have a better idea of what to expect when working in highly variable natural systems.

\section{Statement of Problem}

The problem of interest here is that of the drawdown, as a function of $r, \theta$, and $t$, produced by pumping from a fully penetrating well in the configuration displayed in Figure 1. For the purposes of this development, the pumping well is assumed to be arbitrarily located outside of a circular disk of radius $a$. Flow properties are assumed to be uniform within both the disk and the matrix, although differing between the two. The variation between the disk and matrix can be of any magnitude. Save for the variation of properties between the disk and matrix, the standard conditions for a pumping test in a confined aquifer are assumed at all times. For purposes of this derivation, the origin is assumed to be located at the center of the circular disk. The pumping and observation wells are located at radial distances $r_{p w}$ and $r$, respectively, from the origin. The distance between the pumping and 


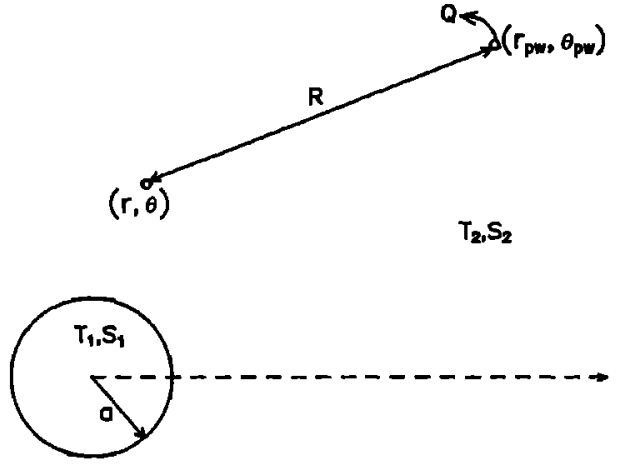

Fig. 1. Nonuniform configuration employed in this work; the center of the disk and the dashed line are the origins for radial and angular locations, respectively; remaining notation explained in the text.

observation wells is defined as $R$ and, from the law of cosines, can be written as

$$
R^{2}=r^{2}+r_{p w}^{2}-2 r r_{p w} \cos \left(\theta-\theta_{p w}\right)
$$

where $\theta_{p w}, \theta$ is the angular position of pumping and observation well, respectively (in radians). Flow within the circular disk $(i=1)$ and surrounding matrix $(i=2)$ can be described mathematically by the polar-coordinate form of the aquifer flow equation:

$$
\frac{\partial^{2} s_{i}}{\partial r^{2}}+\frac{1}{r} \frac{\partial s_{i}}{\partial r}+\frac{1}{r^{2}} \frac{\partial^{2} s_{i}}{\partial \theta^{2}}=\frac{S_{i}}{T_{i}} \frac{\partial s_{i}}{\partial t}
$$

where

$s_{i}$ drawdown in material $i, L$;

$t$ time, $T$;

$S_{i}$ storage coefficient of material $i$ [dimensionless];

$T_{i}$ transmissivity of material $i, L^{2} / T$.

Boundary and initial conditions are as follows:

$$
\begin{gathered}
s_{2}(\infty, \theta, t)=0 \\
s_{i}(r, \theta, 0)=0, \quad 0 \leq r<\infty
\end{gathered}
$$

Note that $s_{1}$ remains finite as $r$ goes to zero:

$$
s_{1}(r, \theta, t)<A, \quad r \rightarrow 0
$$

where

$$
A=f\left(T_{i}, S_{i}, r_{p w}, Q\right)
$$

A pumping well discharging at a constant rate $Q$ is assumed to be at $\left(r_{p w}, \theta_{p w}\right)$, giving rise to the following condition:

$$
\lim _{R \rightarrow 0} 2 \pi R T_{2} \frac{\partial s_{2}(R, t)}{\partial R}=-Q, \quad t>0
$$

In order to ensure continuity of flow between the disk and the matrix, auxiliary conditions at the matrix-disk boundary $(r=a)$ must also be met:

$$
\begin{gathered}
s_{1}(a, \theta, t)=s_{2}(a, \theta, t) \\
T_{1} \frac{\partial s_{1}(a, \theta, t)}{\partial r}=T_{2} \frac{\partial s_{2}(a, \theta, t)}{\partial r}
\end{gathered}
$$

For the purpose of this derivation, we also employ the condition that the drawdown within the matrix $\left(s_{2}\right)$ can be written as the sum of the drawdown predicted by Theis [1935] for pumping in an infinite uniform aquifer $\left(s_{T}\right)$ and the drawdown due to the existence of the circular disk $\left(s_{d}\right)$ :

$$
s_{2}=s_{T}+s_{d}
$$

The Theis drawdown $\left(s_{T}\right)$ can be calculated from (2)-(4) and (6) by neglecting the angular dependence and assuming the origin is at $R=0$ :

$$
s_{T}=\frac{Q}{4 \pi T_{2}} \int_{u_{2}}^{\infty} \frac{\exp (-u)}{u} d u
$$

where

$$
u_{2}=S_{2} R^{2} / 4 T_{2} t
$$

Equation (9) can be substituted into (2) and an analogous equation written solely in terms of $s_{d}$ :

$$
\frac{\partial^{2} s_{d}}{\partial r^{2}}+\frac{\partial s_{d}}{\partial r}+\frac{1}{r^{2}} \frac{\partial^{2} s_{d}}{\partial \theta^{2}}=\frac{S_{2}}{T_{2}} \frac{\partial s_{d}}{\partial t}
$$

The boundary and initial conditions, as well as the auxiliary conditions at the matrix-disk boundary, can also be rewritten in terms of $s_{T}$ and $s_{d}$ :

$$
\begin{gathered}
s_{d}(\infty, \theta, t)=0 \\
s_{1}(r, \theta, 0)=s_{d}(r, \theta, 0)=0, \quad 0 \leq r<\infty \\
s_{I}(a, \theta, t)=s_{d}(a, \theta, t)+s_{T}(R(a, \theta), t) \\
T_{1} \frac{\partial s_{1}(a, \theta, t)}{\partial r}=T_{2} \frac{\partial s_{d}(a, \theta, t)}{\partial r}+T_{2} \frac{\partial s_{T}(R(a, \theta), t)}{\partial r}
\end{gathered}
$$

Equations (2), (5), and (9)-(15) describe the flow conditions of interest here. Appendix A provides the details of the solution derivation. In summary, the approach employs a Laplace transform in time, in conjunction with the method of separation of variables and a modified Bessel function expansion, to obtain functions in transform space that satisfy the transform space analogues of (2), (5), and (9)-(15). The Laplace-space functions that are obtained for the case of a pumping well located outside of the circular pod can be written in nondimensional form as

$$
\begin{array}{r}
\overline{\phi_{1}}\left(\xi, \theta-\theta_{p w^{\prime}}, p\right)=\frac{2 \gamma}{p} \sum_{-\infty}^{\infty} \frac{K_{n}\left(p^{1 / 2} \xi_{p w}\right)}{\Delta_{n}} I_{n}(q \xi) \\
\cdot \cos n\left(\theta-\theta_{p w}\right)
\end{array}
$$

$$
\begin{aligned}
\overline{\phi_{2}} & =\frac{2}{p} \sum_{-\infty}^{\infty} \frac{K_{n}\left(p^{1 / 2} \xi_{p w^{\prime \prime}}\right)}{\Delta_{n}}\left[I_{n}\left(p^{1 / 2} \xi\right) \Delta_{n}\right. \\
& \left.+\Theta_{n} K_{n}\left(p^{1 / 2} \xi\right)\right] \cos n\left(\theta-\theta_{p w^{\prime}}\right), \quad \xi<\xi_{p w}
\end{aligned}
$$




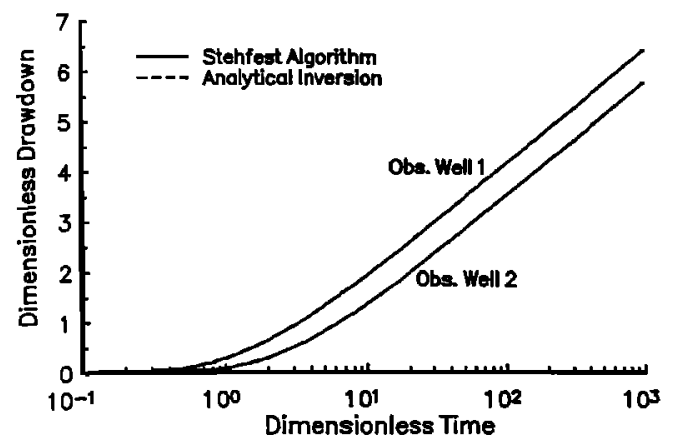

Fig. 2. Dimensionless drawdown $\left(\left(4 \pi T_{2} s_{i}\right) / Q\right)$ versus time $\left(T_{2} t / S_{2} a^{2}\right)$ plot comparing the results of the analytical inversion of (16) and (17) (see Appendix B) with those from the numerical inversion of the same equations using the Stehfest algorithm (observation well 1 located at $\xi(r / a)=0.4, \theta=1.05 \mathrm{rad}$, observation well 2 located at $\xi=3.6, \theta=2.09 \mathrm{rad}$, pumping well located at $\xi_{p w^{\prime}}=6, \theta=0 \mathrm{rad} ; \gamma=\rho=10$ ).

$$
\begin{aligned}
\overline{\phi_{2}} & =\frac{2}{p} \sum_{-\infty}^{\infty} \frac{K_{n}\left(p^{1 / 2} \xi\right)}{\Delta_{n}}\left[I_{n}\left(p^{1 / 2} \xi_{p w}\right) \Delta_{n}\right. \\
& \left.+\Theta_{n} K_{n}\left(p^{1 / 2} \xi_{p w}\right)\right] \cos n\left(\theta-\theta_{p w}\right), \quad \xi>\xi_{p w}
\end{aligned}
$$

where

$\bar{\phi}_{i} \quad$ Laplace transform of $\phi_{i}$, equal to $\int_{0}^{\infty} e^{-p \tau} \phi_{i} d \tau$; $\phi_{i}=\left(4 \pi T_{2} / Q\right) s_{i}$

$p$ Laplace-transform variable, complex;

$\xi=r / a$

$\xi_{p w}=r_{p w} / a$

$\tau=T_{2} t / a^{2} S_{2}$

$\gamma=T_{2} / T_{1}$;

$I_{n}$ modified Bessel function of the first kind of order $n$;

$K_{n}$ modified Bessel function of the second kind of order $n$;

$\Delta_{n}=q I_{n}^{\prime}(q) K_{n}\left(p^{1 / 2}\right)-p^{1 / 2} \gamma K_{n}^{\prime}\left(p^{1 / 2}\right) I_{n}(q) ;$

$\Theta_{n}=\gamma p^{1 / 2} I_{n}(q) I_{n}^{\prime}\left(p^{1 / 2}\right)-q I_{n}\left(p^{1 / 2}\right) I_{n}^{\prime}(q)$;

$q=(\rho p)^{1 / 2}$;

$\rho=\left(S_{1} / T_{1}\right)\left(T_{2} / S_{2}\right)$.

For equations of the complexity of (16) and (17), the analytical back transformation from Laplace space is quite tedious and requires computer-intensive numerical integration. Therefore, for most applications, the back transformation is best performed numerically. An algorithm developed by Stehfest [1970], which has been found to be of great use in well hydraulics applications [e.g., Moench and Ogata, 1984], was employed here to perform the numerical Laplace inversion. In order to demonstrate the viability of the Stehfest algorithm for this specific application, the analytical inversion of (16) and (17) was also performed, and the analytical and numerical results compared. Appendix $B^{1}$ briefly outlines the analytical inversion for (16). Figure 2 displays the results of a comparison of the analytical and Stehfest inversion schemes for observation wells located both within and outside of the circular pod. In both cases,

\footnotetext{
${ }^{1}$ Appendices $B$ and $C$ are available with entire article on microfiche. Order from American Geophysical Union, 2000 Florida Avenue, N. W., Washington, DC 20009. Document W92-003; $\$ 2.50$. Payment must accompany order.
}

the analytical and numerical results are essentially indistinguishable from one another. Therefore, because of its much greater efficiency and acceptable accuracy, the Stehfest algorithm is employed to perform the back transformations for the remainder of this work.

\section{Sensitivity Analysis of Theoretical Responses}

The primary motivation of this work was to develop greater insight into the nature of pumping induced drawdown in nonuniform aquifers. In this section, the technique of sensitivity analysis [McElwee and Yukler, 1978; McElwee, 1987] is employed to help develop an understanding of the relationship between drawdown and flow properties in the nonuniform configuration considered here. The sensitivity of drawdown at $(r, \theta, t)$ to changes in a given parameter such as the transmissivity of the disk $\left(\partial s_{i}(r, \theta, t) / \partial T_{1}\right)$ is termed a sensitivity coefficient. The multiplication of a sensitivity coefficient by the parameter of interest is defined as a normalized sensitivity coefficient [McElw'ee, 1987]. The normalized sensitivity coefficient is utilized in the discussions of this work to examine the dependence of drawdown on flow properties of the circular disk.

Figure 3 depicts the results of an analysis of the sensitivity of drawdown at an observation well to the properties of the circular disk. Curves a-d show the sensitivity of drawdown to the transmissivity of the disk for observation wells located both within and outside of the disk. Note that for an observation well located at the center of the disk (curve a), the sensitivity curve reaches a peak and then falls quickly to zero. Thus, in this particular case, there is only a short interval of time during which information can be gained about the transmissive properties of the disk. After that time has passed, drawdown is completely independent of disk transmissivity. For observation wells located elsewhere in the disk, the sensitivity plot will be similar to that of curve $b$, which is positive at early times and negative at large times. The positive sensitivity is produced as the frontal portion of the pumping-induced pressure disturbance passes the observation well. After passage of the pressure front, the sensitivity becomes negative and flattens to a constant value.

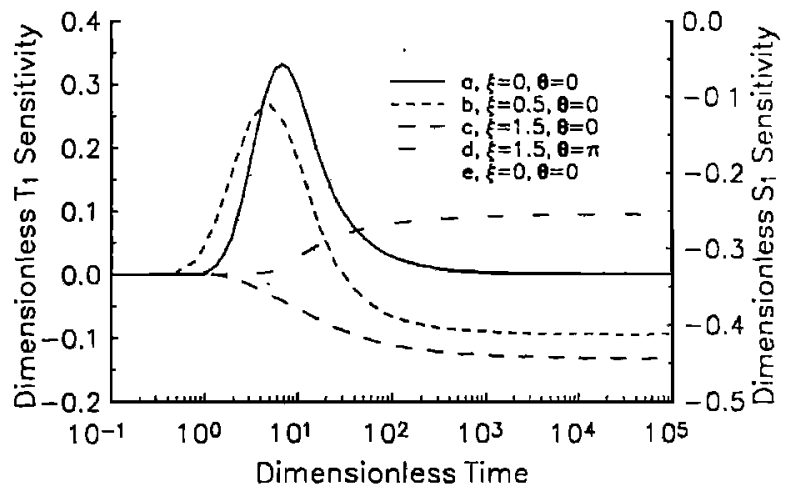

Fig. 3. Dimensionless sensitivity to disk properties versus time $\left(T_{2} t / S_{2} a^{2}\right)$ plot. Curves a-d depict dimensionless normalized sensitivity to disk transmissivity $\left(T_{1} \partial\left[\left(4 \pi T_{2} s_{i}\right) / Q\right] / a T_{1}\right)$ and curve e depicts sensitivity to disk storativity $\left(S_{1} \partial\left[\left(4 \pi T_{2} s_{1}\right) / Q\right] / \partial S_{1}\right)$ (pumping well located at $\xi_{p w}=6, \theta=0$ rad; $\gamma=\rho=10$ ). Note that each curve is defined according to the radial and angular position of the observation well. 
Regardless of observation-well location, however, there is only a finite interval of time during which the sensitivity to disk transmissivity is changing. After that interval, the sensitivity relationships remain constant with time, indicating changes in drawdown are independent of disk transmissivity. Butler and McElwee [1990] show that data gathered during a period of constant sensitivity will be essentially of no use for describing aquifer nonuniformities. Data collection during the period of changing sensitivities is required if information is to be gained about flow properties in a particular portion of an aquifer. Although their study employed a radially symmetric nonuniform configuration, the relationships displayed in Figure 3 show that the conclusions of Butler and McElwee [1990] extend to the radially asymmetric case as well.

Curves $c$ and $d$ of Figure 3 indicate that drawdown at observation wells in the vicinity of the disk will also display some sensitivity to disk transmissivity. However, the region of the matrix in which measurable drawdown will reflect the existence of the disk is quite limited. Figure 4 is an areal view of pumping-induced drawdown in an aquifer in which a disk of anomalous properties is located at a distance from the pumping well. The limited angular and radial region in which the disk has a significant impact on pumping-induced drawdown is clearly illustrated. Note that the contour interval in Figure 4 is finer in the vicinity of the disk in order to better depict the curvature of the drawdown contour lines produced by the embedded disk.

Curve e of Figure 3 is a general depiction of the sensitivity of drawdown to the storativity of the disk. In all cases, there is only a finite duration of time during which both changes in drawdown and total drawdown are dependent on the storativity of the disk. After that time, no information about the storage properties of the disk can be gained from drawdown measurements.

The sensitivity relationships for both the transmissivity and storativity of the disk indicate the importance of data collection during the early time period when the sensitivity relationships are changing. Without collection of highquality data at a high frequency during this period, there is little hope of learning much about the spatial variations in

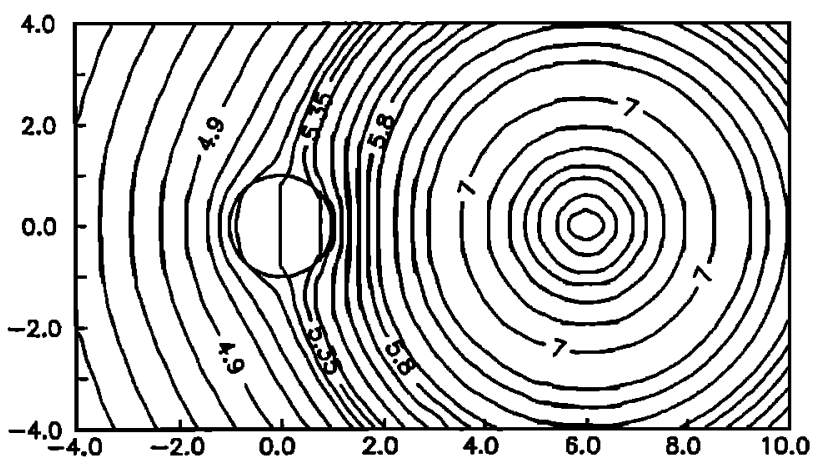

Fig. 4. Areal view of dimensionless pumping-induced drawdown $\left(4 \pi T_{2} s_{1} / Q\right)$ at a dimensionless time $\left(T_{2} t / S_{2} a^{2}\right)$ of 3200 ; contour interval varies $(0.15$ from 4.30 to $5.05,0.10$ from 5.05 to $5.25,0.05$ from 5.25 to $5.40,0.10$ from 5.40 to $5.90,0.20$ from 5.90 to $6.50,0.50$ from 6.50 to 9.00 , and 1.0 from 9.00 to 11.00 ) in order to emphasize the effect of the disk of anomalous properties $(\gamma=\rho=$ $10)$. Note that the origin is at the center of the disk and distances are given in $\xi(r / a)$.

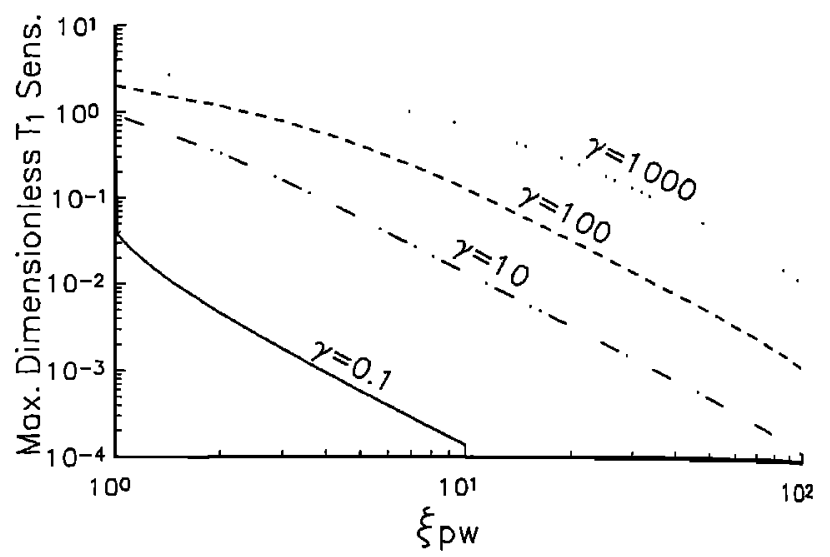

Fig. 5. Dimensionless maximum normalized sensitivity to disk transmissivity $\left(T_{1} \partial\left[\left(4 \pi T_{2} s_{1}\right) / Q\right] / \partial T_{1}\right)$ versus dimensionless distance to the pumping well $\left(r_{p w} / a\right)$ plot for different transmissivity contrasts (observation well located at $\xi(r / a)=0, \theta=0 \mathrm{rad} ; \theta_{p w}=$ $0 \mathrm{rad} ; \gamma=\rho$ ).

aquifer flow properties using a conventional constant rate pumping test. Butler and McElwee [1990] suggest a variable rate pumping strategy in order to obtain multiple samples of the critical early time data and to decrease the correlation between the effects of flow properties in different portions of the aquifer. Even this scheme, however, will probably only be appropriate for characterizing very large scale variations in flow properties.

Figure 3 illustrates the dependence of drawdown on disk properties for a disk located at a distance of $\xi_{p w}=6$ from the pumping well. Although the form of the sensitivity relationships does not change with radial location of the disk, the magnitude of the sensitivity will change greatly. Figure 5 depicts changes in the maximum sensitivity of drawdown at the center of the disk to disk transmissivity (peak value of curve a of Figure 3 ) as a function of the radial distance of the disk from the pumping well. Results for four different cases are shown in order to illustrate behavior for a range of transmissivity contrasts. In all cases, there is a dramatic decrease of sensitivity with radial distance from the pumping well. At a large distance from the pumping well (distance beyond which a curve in Figure 5 is linear), the properties of the disk have essentially no influence on drawdown at an observation well sited within the disk. Thus, if there is a zone of low permeability material in the vicinity of an observation well, the effect of that zone on drawdown measurements at the observation well will be negligible for observation wells a considerable distance from the pumping well, a result in keeping with the findings of Tongpenyai and Raghavan [1981] concerning the effect of an infinitely thin skin on observation-well drawdown. Butler [1990] uses the solution developed here to illustrate how log-log drawdown versus time curves converge on the uniform-aquifer curve as the distance between the disk and the pumping well increases for the case of an observation well located in the vicinity of a zone of low permeability.

The dimensionless relationships depicted in Figure 5 clearly indicate that the volume of the aquifer that is controlling a given increment of observation-well drawdown increases in size as observation wells at greater distances from the pumping well are considered. In order for disks at different radial positions to have the same influence on 
drawdown at an observation well, $\xi_{p w}$ must remain constant. Thus a disk located at $100 \mathrm{~m}$ from the pumping well must be $25 \mathrm{~m}$ in radius in order to have the same influence on drawdown as a disk of $1 \mathrm{~m}$ in radius located at four meters from the pumping well. Given the very large aquifer volumes that are controlling observation-well responses at moderate to large distances from the pumping well, it is no surprise that conventional methods of pumping test analysis, which assume constant aquifer properties, have proven so successful in field investigations. Except in the case of quite large-scale variations in flow properties, the size of the aquifer volume controlling drawdown will be large enough to essentially filter out the natural property variations in a unit. Thus good fits between the ideal models of the wellhydraulics literature [e.g., Theis, 1935; Hantush, 1964; etc.] and measured field data should be the rule, not the exception, when observation wells at a distance from the pumping well are employed.

An issue with considerable practical ramifications is that of the variations that might be expected between pumpingtest parameters calculated from drawdown at different observation wells. Butler [1991] addresses this issue in a Monte Carlo analysis of pumping tests in nonuniform aquifers whose transmissivity variations can be described by secondorder stationary stochastic processes. His findings concerning radial, angular, and temporal variations in estimated transmissivity can be simply explained using the relationships depicted in Figure 5. A Theis analysis of drawdown from observation wells at differing radial distances from the pumping well would be expected to produce a set of radially dependent transmissivity estimates (at least in the vicinity of the pumping well) because of the difference in the size of the aquifer volumes controlling the early-time response data. If the wells were located at the same radial distance from the pumping well but at differing angular positions, one would expect much smaller differences between the estimated parameters because of the similar sizes of the volumes controlling the response data. In all cases, if drawdown at large distances from the pumping well or changes of drawdown at large dimensionless times were employed in the analysis, one would expect to see little if any difference between the estimated parameters because of the high degree of correlation between the very large aquifer volumes controlling observation-well drawdown at large distances and times. Thus it will be difficult to gain much information about the variation of flow properties in the subsurface using a constant rate pumping test with many observation wells.

\section{LARge Time Approximations OF THEORETICAL RESPONSES}

An important advantage of an analytical solution over its numerical counterpart is that the functional form of the analytical solution can often allow some insight to be gained into the processes governing solution behavior. Unfortunately, the complexity of (16) and (17) makes it difficult to obtain much insight through direct examination of the equations. Large-time approximations of these equations, however, can be developed so that additional insight into the controls on drawdown during a pumping test can be gained. These approximate forms can be derived by developing equations in Laplace space that are valid approximations for small arguments, and then analytically inverting these equa- tions to real space. The spectral representation of the inversion integral shows that the small-argument equations in Laplace space are equivalent to large-time expressions in real space. Small arguments in Laplace space represent low frequency components and thus are appropriate for consideration of large-time behavior.

The large-time expressions for (16) and (17a) are as follows:

$$
\phi_{1}=\ln \left(\frac{4 \tau}{C \xi_{p w}^{2}}\right)+\frac{4 \gamma}{\gamma+1} \sum_{n=1}^{x} \frac{1}{n}\left(\frac{\xi}{\xi_{p w}}\right)^{n} \cos n\left(\theta-\theta_{p w}\right)
$$

$$
\begin{aligned}
\phi_{2} & =\ln \left(\frac{4 \tau}{C \xi_{p w^{\prime}}^{2}}\right)+2 \sum_{n=1}^{\infty} \frac{1}{n}\left[\left(\frac{\xi}{\xi_{p w^{\prime}}}\right)^{n}\right. \\
& \left.+\left(\frac{1}{\xi \xi_{p w}}\right)^{n}\left(\frac{\gamma-1}{\gamma+1}\right)\right] \cos n\left(\theta-\theta_{p w}\right), \quad \xi<\xi_{p w}
\end{aligned}
$$

Where $C=\exp \left(\gamma^{*}\right), \gamma^{*}$ is Euler's constant $(0.5772 \cdots)$. For the case of $\xi>\xi_{p w}, \xi$ and $\xi_{p w}$ are interchanged in (19). Appendix $\mathrm{C}$ (on microfiche) details the derivation of these expressions. Note that in all cases the small-argument approximations are equivalent to the $u<0.01$ condition used in the truncation of the infinite series representation of the well function of Theis [1935]. This equivalence results from the physical significance of the infinite series truncation as discussed by Butler [1988].

The form of (18) and (19) provides considerable information concerning the nature of pumping induced drawdown in nonuniform aquifers. Both equations indicate that, at large times, changes in drawdown at an observation well are solely dependent on the transmissivity of the matrix and the rate of pumpage (the two parameters used to normalize drawdown). The properties of the disk have no impact on changes in drawdown at large times. Thus the semilog method of Cooper and Jacob [1946] for estimation of flow properties in uniform aquifers can also be employed in this case to estimate matrix transmissivity. When coupled with the findings of earlier analytical studies of pumping-induced drawdown in nonuniform aquifers [e.g., Barker and Herbert, 1982; Butler and Liu, 1991], this result indicates that the semilog method for drawdown analysis will be viable in any laterally nonuniform system if the flow to the pumping well is approximately radial during the period of the analysis. The criterion equivalent to the $u<0.01$ condition, however, will differ depending on the particular type of laterally nonuniform system.

Consideration of the individual equations allows additional conclusions to be drawn concerning pumping-induced drawdown in nonuniform aquifers. Equation (18) consists of two terms: the first term considers drawdown in a uniform aquifer at a distance from the pumping well equal to the distance between the pumping well and the center of the disk, and the second term considers the impact of the disk, correcting for the fact that the observation point may not be at the center of the disk. The magnitude of the second term depends on the position of the observation well within the disk, with the maximum magnitude occurring when an observation well is located at the disk edge. Note that, as shown by the sensitivity analysis, large-time drawdown at an 
Models of Lateral Heterogeneities

1. Butler et ol. (1989)

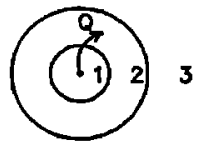

2. Butler and Lu (this article)

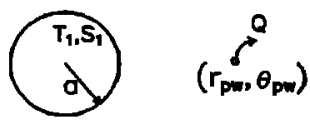

3. Butler and Lu (1991)

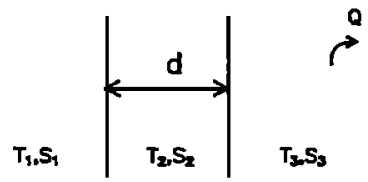

Fig. 6. Several models of lateral heterogeneities in aquifer properties for which analytical solutions have been developed.

observation well located at the center of the disk is independent of disk size and properties. Thus early-time data are often necessary to detect the existence of a zone of anomalous properties. However, as shown in Figure 5, the impact of disk properties on early-time drawdown becomes negligible as the distance from the pumping well increases. Also note that when the cosine term equals one (angular positions of observation and pumping wells are the same) and $\gamma$ equals one, the second term converges to a form equivalent to the Theim equation [Bear, 1979], an expected result given that the first term is the drawdown at a radial distance of $\xi_{p w}$ from the pumping well.

Equation (19) is similar to (18) in that the first term considers drawdown in a uniform aquifer at a distance from the pumping well equal to the distance between the pumping well and the center of the disk. The second term considers the impact of the disk and corrects for the fact that the observation well is not in the disk in this case. As in (18), the drawdown due to the disk goes to zero as the disk increases in distance from the pumping well. The only correction in that case is a function of the distance between the observation well and the disk (i.e., an expression equivalent to the Theim equation). The impact of the disk is primarily a function of its size, relative to the location of the observation and pumping wells, and the transmissivity contrast. As would be expected, the smaller the disk and the smaller the transmissivity contrast, the less impact the disk has on observation-well drawdown.

\section{SummaRY AND CONClusions}

This article has presented an analytical solution for pumping-induced drawdown in a nonuniform aquifer that consists of a disk of anomalous properties arbitrarily located in a uniform media. When considered in conjunction with the solution of Jaeger [1944], this work allows the effects of a pumping well located either within or outside of the disk to be assessed.

The focus of this work, however, was not on the details of the solution methodology but rather on the use of this solution to develop insight into the effects of lateral hetera geneities on pumping-induced drawdown. Sensitivity analy. sis and large-time approximations of the solution were used here to examine how drawdown at an observation well is influenced by a disk of properties dissimilar from those of the enclosing matrix.

Regardless of observation-well placement, the transmissivity of the disk influences changes in drawdown for a time of quite limited duration. Although total drawdown may always be sensitive to disk transmissivity, it is virtually impossible to gain more information about the transmissivity of the disk once changes in drawdown are independent of disk transmissivity. In the case of disk storativity, there is a finite interval of time during which not only changes in drawdown but also total drawdown are dependent on the storativity of the disk. After that time, no information about the storativity of the disk can be obtained from drawdown measurements.

The influence of disk properties on observation-well drawdown is heavily dependent on the radial distance between the disk and the pumping well. As the disk increases in distance from the pumping well, its influence on observationwell drawdown decreases dramatically. At observations wells located at moderate to large distances from the pumping well, the size of the aquifer volume controlling even early-time drawdown is quite large. The large size of this controlling volume effectively filters out the influence of all but the very large scale variations in flow properties from observation-well drawdown. Thus, in general, drawdown data from wells located at a distance from the pumping well should nicely fit the ideal uniform aquifer models of the well hydraulics literature. Note that the conclusions of this analysis should not be considered limited to the study of circular disks, as these findings should be appropriate for a patch of material of any arbitrary shape enclosed in a matrix of differing properties.

The work described in this article completes a series of papers on the effects of lateral heterogeneities on drawdown during constant rate pumping tests. Butler [1991] presented a Monte Carlo analysis of the effects of lateral heterogeneities on pumping-induced drawdown. Although results concerning the dependence of the transmissivity estimated from a pumping test on the radial, angular, and temporal position of the observations were obtained, relatively little insight could be gained concerning the controls on drawdown in nonuniform systems. In order to gain more insight into conditions in nonuniform systems, analytical solutions for pumpinginduced drawdown in idealized nonuniform aquifers were developed (see Figure 6). These idealized configurations were designed so that the effects of different nonuniform structures on drawdown could be isolated. When considered together, the results from this series of analytical solutions provide considerable insight into pumping-induced drawdown in nonuniform systems.

In all cases, regardless of the specifics of the particular configuration, the following statements can be made about pumping tests in nonuniform aquifers. First, changes in pumping-induced drawdown will be dependent on the properties of a discrete portion of the aquifer for a time of quite 
limited duration. After that time, it will be difficult to gain further information concerning the properties of that portion of the aquifer from drawdown measurements. Thus, a technique that uses changes in drawdown to estimate the transmissivity of an aquifer (such as the Cooper-Jacob semilog method) can often be configured so that the influence of properties in a certain portion of the system (e.g., a near-well zone of disturbance) can be removed. Second, there is considerable correlation between the effects of hydraulic properties in different portions of an aquifer on drawdown. Unless this correlation can be decreased, it will be very difficult to reliably estimate the nature of spatial variations in flow properties. Third, the radial location of an observation well is critical for characterizing spatial variations in flow properties. If an observation well is located at a considerable distance from the pumping well, the volume of the aquifer controlling drawdown at that well is so large that it will be difficult to detect the existence of spatial variations in flow properties. Even an observation well close to the pumping well, however, can only provide limited information about spatial variations in aquifer properties. Fourth, drawdown at an observation well is rather weakly dependent on the properties of material between the observation and pumping wells. Generally, only small-time data are of use for estimation of these interwell properties. Thus special attention must be given to data collection strategies and equipment that will allow large amounts of high-quality data to be collected during the early-time period.

The overall conclusion of this body of work is that constant rate pumping tests are not a very effective tool for characterizing lateral variations in flow properties. If information about lateral variations in flow properties is to be obtained from hydraulic tests, variable rate pumping strategies, such as the pulse test of Johnson et al. [1966] and the variable rate test proposed by Butler and McElwee [1990], or a program of multiple slug tests must be pursued. Further work, however, is required to fully assess those approaches for characterization of flow properties in nonuniform systems.

\section{APPENDIX A}

In this section, the mathematical derivation of the solution discussed in the main body of the article is presented. For the sake of generality, the solution is obtained in a dimensionless form. The approach used here is an extension of that employed by Jaeger [1944]. First, the Laplace transformation technique [Carslaw and Jaeger, 1959; Doetsch, 1961] is applied to remove the time dependence from the equations. The resulting partial differential equations in terms of radial distance and $\theta$ are solved using separation of variables [Haberman, 1987]. The solution constants are then evaluated using boundary conditions and the orthogonality of cosine and sine functions. After the derivation of a solution in Laplace space has been presented, the solution is verified by reduction to a simpler form for which a solution has previously been found. The section concludes with a brief discussion of the numerical inversion technique employed here.

In this derivation, a solution is presented for the case of drawdown as a result of a pumping well located outside of the circular disk. Equations (2), (11), (5), (9), (10), and (12)-(15) describe the flow conditions of interest here. To work with the most general form of the solution, this derivation is performed using the following dimensionless forms of (2), (11), (5), (9), (10), and (12)-(15):

$$
\begin{gathered}
\frac{\partial^{2} \phi_{1}}{\partial \xi^{2}}+\frac{1}{\xi} \frac{\partial \phi_{1}}{\partial \xi}+\frac{1}{\xi^{2}} \frac{\partial^{2} \phi_{1}}{\partial \theta^{2}}=\rho \frac{\partial \phi_{1}}{\partial \tau}, \quad 0 \leq \xi \leq 1 \\
\frac{\partial^{2} \phi_{d}}{\partial \xi^{2}}+\frac{1}{\xi} \frac{\partial \phi_{d}}{\partial \xi}+\frac{1}{\xi^{2}} \frac{\partial^{2} \phi_{d}}{\partial \theta^{2}}=\frac{\partial \phi_{d}}{\partial \tau}, \quad 1 \leq \xi<\infty \\
\phi_{1}(\xi, \theta, \tau)<A^{\prime}, \quad \xi \rightarrow 0 \\
\phi_{2}=\phi_{T}+\phi_{d} \\
\phi_{T}=\int_{u_{2}}^{\infty} \frac{\exp (-u)}{u} d u \\
\phi_{d}(\xi, \theta, \theta, \tau)=0 \\
\phi_{1}(1, \theta, \tau)=\phi_{d}(1, \theta, \tau)+\phi_{T}\left(\xi_{R}(1, \theta), \tau\right) \\
\left.\frac{\partial \phi_{1}(1, \theta, \tau)}{\partial \xi}=\gamma \frac{\partial \phi_{d}(1, \theta, \tau)}{\partial \xi}+\gamma \frac{\partial \phi_{T}\left(\xi_{R}(1, \theta)\right.}{\partial \xi}, \tau\right)
\end{gathered}
$$

where

$$
\begin{aligned}
\phi_{i} & =\left(4 \pi T_{2} / Q\right) s_{i} ; \\
\xi & =r / a ; \\
\xi_{R} & =R / a ; \\
\tau & =T_{2} t / a^{2} S_{2} ; \\
\gamma & =T_{2} / T_{1} ; \\
\rho & =\left(S_{1} / T_{1}\right)\left(T_{2} / S_{2}\right) .
\end{aligned}
$$

The Laplace-space analogues to (A1)-(A9) are as follows:

$$
\frac{\partial^{2} \overline{\phi_{1}}}{\partial \xi^{2}}+\frac{1}{\xi} \frac{\partial \overline{\phi_{1}}}{\partial \xi}+\frac{1}{\xi^{2}} \frac{\partial^{2} \overline{\phi_{1}}}{\partial \theta^{2}}-q^{2} \overline{\phi_{1}}=0,
$$

$$
0 \leq \xi \leq 1
$$

$$
\frac{\partial^{2} \overline{\phi_{d}}}{\partial \xi^{2}}+\frac{1}{\xi} \frac{\partial \overline{\phi_{d}}}{\partial \xi}+\frac{1}{\xi^{2}} \frac{\partial^{2} \overline{\phi_{d}}}{\partial \theta^{d}}-p \overline{\phi_{d}}=0
$$

$$
1 \leq \xi<\infty
$$

$$
\begin{gathered}
\overline{\phi_{1}}(\xi, \theta, p)<A^{\prime} / p, \quad \xi \rightarrow 0 \\
\overline{\phi_{2}}(\xi, \theta, p)=\overline{\phi_{d}}(\xi, \theta, p)+\overline{\phi_{T}}\left(\xi_{R}(\xi, \theta), p\right) \\
\overline{\phi_{T}}(\xi, p)=\frac{2}{p} K_{0}\left(p^{1 / 2} \xi_{R}\right) \\
\overline{\phi_{d}}(\infty, \theta, p)=0 \\
\overline{\phi_{1}}(1, \theta, p)=\overline{\phi_{d}}(1, \theta, p)+\overline{\phi_{T}}\left(\xi_{R}(1, \theta), p\right)
\end{gathered}
$$

$$
\frac{\partial \overline{\phi_{1}}(1, \theta, p)}{\partial \xi}=\gamma \frac{\partial \overline{\phi_{d}}(1, \theta, p)}{\partial \xi}+\gamma \frac{\partial \overline{\phi_{T}}\left(\xi_{R}(1, \theta), p\right)}{\partial \xi}
$$


where

$\bar{\phi}_{i}=$ Laplace transform of $\phi_{i}$, equal to $\int_{0}^{\infty} e^{-p \tau} \phi_{i} d \tau$; $q=(\rho p)^{1 / 2}$;

p Laplace-transform variable, complex;

$K_{0}$ modified Bessel function of the second kind of order zero.

Jaeger [1944] notes that a modified Bessel function of the second kind of order zero with an argument $\xi_{R}$ can be expanded as an infinite series of cosine terms and modified Bessel functions in the following manner:

$$
\begin{gathered}
\overline{\phi_{T}}\left(\xi_{R}, p\right)=\frac{2}{p} K_{0}\left(p^{1 / 2} \xi_{R}\right) \\
=\overline{\phi_{T}}(\xi, \theta, p)=\frac{2}{p} \sum_{-\infty}^{\infty} \cos n\left(\theta-\theta_{p w}\right) \\
\cdot I_{n}\left(p^{1 / 2} \xi\right) K_{n}\left(p^{1 / 2} \xi_{p w}\right), \quad \xi<\xi_{p w} \\
\overline{\phi_{T}}\left(\xi_{R}, p\right)=\frac{2}{p} K_{0}\left(p^{1 / 2} \xi_{R}\right) \\
=\overline{\phi_{T}}(\xi, \theta, p)=\frac{2}{p} \sum_{-\infty}^{\infty} \cos n\left(\theta-\theta_{p w}\right) \\
\cdot I_{n}\left(p^{1 / 2} \xi_{p w}\right) K_{n}\left(p^{1 / 2} \xi\right), \quad \xi_{p w}<\xi
\end{gathered}
$$

where $\xi_{p w}=r_{p w} / a ; I_{n}$ is a modified Bessel function of the first kind of order $n$; and $K_{n}$ is a modified Bessel function of the second kind of order $n$. The proof of this expansion can be found in the discussion of Gray et al. [1952] regarding the addition theorems for Bessel functions [cf. Gray et al., 1952, p. 74].

This expansion allows conditions (A13), (A16) and (A17) to be rewritten:

$$
\begin{gathered}
\overline{\phi_{2}}(\xi, \theta, p)=\overline{\phi_{d}}(\xi, \theta, p)+\overline{\phi_{T}}(\xi, \theta, p) \\
\overline{\phi_{1}}(1, \theta, p)=\overline{\phi_{d}}(1, \theta, p)+\overline{\phi_{T}}(1, \theta, p) \\
\frac{\partial \overline{\phi_{1}}(1, \theta, p)}{\partial \xi}=\gamma \frac{\partial \overline{\phi_{d}}(1, \theta, p)}{\partial \xi}+\gamma \frac{\partial \overline{\phi_{T}}(1, \theta, p)}{\partial \xi}
\end{gathered}
$$

Given conditions (A12), (A15), and (A18)-(A21), solutions can be obtained for (A10) and (A11) using separation of variables.

The basic approach is to assume a solution for (A10) and (A11) can be written as:

$$
\overline{\phi_{i}}=G(\theta) F(\xi)
$$

Substitution of (A22) into (A10) and rearrangement produces the following set of equations:

$$
\begin{gathered}
G^{\prime \prime}(\theta)=-\lambda G(\theta) \\
\xi^{2} F^{\prime \prime}(\xi)+\xi F^{\prime}(\xi)-\left(\xi^{2} q^{2}+\lambda\right) F(\xi)=0
\end{gathered}
$$

where $\lambda$ is a separation constant.

Equation (A23a) is a simple ordinary differential equation with the following general solution:

$$
G(\theta)=C_{1} \cos \left(\lambda^{1 / 2} \theta\right)+C_{2} \sin \left(\lambda^{1 / 2} \theta\right)
$$

Given periodic boundary conditions,

$$
\begin{gathered}
G(-\pi)=G(\pi) \\
G^{\prime}(-\pi)=G^{\prime}(\pi),
\end{gathered}
$$

evaluation of (A24) at the boundaries produces the following condition:

$$
\sin \left(\lambda^{1 / 2} \pi\right)=0
$$

Thus

$$
\lambda=n^{2}, \quad n=0, \pm 1,2, \cdots
$$

and (A24) can be rewritten:

$$
\begin{array}{r}
G(\theta)=C_{1_{n}} \cos (n \theta)+C_{2_{n}} \sin (n \theta), \\
n=0, \pm 1,2 \cdots
\end{array}
$$

The solution to (A23b) is also straightforward as (A23b) can be rewritten as a modified Bessel equation with the following general solution:

$$
\begin{array}{r}
F(\xi)=C_{3_{n}} K_{n}(q \xi)+C_{4_{n}} I_{n}(q \xi), \\
n=0, \pm 1,2, \cdots
\end{array}
$$

Since $\bar{\phi}_{1}$ is finite as $\xi$ goes to zero (see (A12)), (A26) can be reduced to

$$
F(\xi)=C_{4_{n}} I_{n}(q \xi), \quad n=0, \pm 1,2, \cdots
$$

The general solution to (A10) can then be written as the product of (A25) and (A27):

$$
\begin{aligned}
\overline{\phi_{1}}\left(\xi, \theta-\theta_{p w}, p\right) & =\sum_{-\infty}^{\infty} C_{1 n} I_{n}(q \xi) \cos n\left(\theta-\theta_{p w}\right) \\
& +\sum_{-\infty}^{\infty} C_{2 n} I_{n}(q \xi) \sin n\left(\theta-\theta_{p w}\right)
\end{aligned}
$$

where the angular argument is written in terms of $\left(\theta-\theta_{p w}\right)$ in keeping with (A18). The general solution to (A11) can be found in an analogous manner using condition (A15) instead of (A12):

$$
\begin{aligned}
\overline{\phi_{d}}\left(\xi, \theta-\theta_{p w}, p\right) & =\sum_{-\infty}^{\infty} C_{3 n} K_{n}\left(p^{1 / 2} \xi\right) \cos n\left(\theta-\theta_{p w}\right) \\
& +\sum_{-\infty}^{\infty} C_{4 n} K_{n}\left(p^{1 / 2} \xi\right) \sin n\left(\theta-\theta_{p w}\right)
\end{aligned}
$$

In order to evaluate the constants $\left(C_{\text {in }}\right),(\mathrm{A} 18 a),(\mathrm{A} 28)$, and (A29) are substituted into (A20) and (A21):

$$
\begin{aligned}
& \sum_{-\infty}^{\infty} C_{1 n} I_{n}(q) \cos n\left(\theta-\theta_{p w}\right) \\
& \quad+\sum_{-\infty}^{\infty} C_{2 n} I_{n}(q) \sin n\left(\theta-\theta_{p w}\right)
\end{aligned}
$$




$$
\begin{aligned}
& =\frac{2}{p} \sum_{-\infty}^{\infty} I_{n}\left(p^{1 / 2}\right) K_{n}\left(p^{1 / 2} \xi_{p w}\right) \cos n\left(\theta-\theta_{p w}\right) \\
& +\sum_{-\infty}^{\infty} C_{3 n} K_{n}\left(p^{1 / 2}\right) \cos n\left(\theta-\theta_{p w^{\prime}}\right) \\
& +\sum_{-\infty}^{\infty} C_{4 n} K_{n}\left(p^{1 / 2}\right) \sin n\left(\theta-\theta_{p w}\right) \\
& q\left[\sum_{-\infty}^{\infty} C_{1 n} I_{n}^{\prime}(q) \cos n\left(\theta-\theta_{p w}\right)\right. \\
& \left.+\sum_{-x}^{\infty} C_{2 n} I_{n}^{\prime}(q) \sin n\left(\theta-\theta_{p w}\right)\right] \\
& =\frac{2 \gamma}{p^{1 / 2}} \sum_{-\infty}^{\infty} I_{n}^{\prime}\left(p^{1 / 2}\right) K_{n}\left(p^{1 / 2} \xi_{p w}\right) \\
& \cdot \cos n\left(\theta-\theta_{p w}\right)+p^{1 / 2} \gamma \\
& \cdot\left[\sum_{-\infty}^{\infty} C_{3 n} K_{n}^{\prime}\left(p^{1 / 2}\right) \cos n\left(\theta-\theta_{p n^{\prime}}\right)\right. \\
& \left.+\sum_{-\infty}^{\infty} C_{4 n} K_{n}^{\prime}\left(p^{1 / 2}\right) \sin n\left(\theta-\theta_{p w}\right)\right]
\end{aligned}
$$

$$
\begin{gathered}
=\frac{2 \gamma}{p} \frac{K_{m}\left(p^{1 / 2} \xi_{p w^{\prime}}\right)}{\Delta_{m}} \\
C_{3 m}=\frac{2}{\Delta_{m} p}\left(\frac{\gamma I_{m}(q)}{K_{m}\left(p^{1 / 2}\right)}-\frac{\Delta_{m} I_{m}\left(p^{1 / 2}\right)}{K_{m}\left(p^{1 / 2}\right)}\right) K_{m}\left(p^{1 / 2} \xi_{p w}\right) \\
=\frac{2}{p} \frac{\Theta_{m}}{\Delta_{m}} K_{m}\left(p^{1 / 2} \xi_{p w}\right)
\end{gathered}
$$

where

$$
\begin{aligned}
& \Delta_{m}=q I_{m}^{\prime}(q) K_{m}\left(p^{1 / 2}\right)-p^{1 / 2} \gamma K_{m}^{\prime}\left(p^{1 / 2}\right) I_{m}(q) \\
\Theta_{m}= & \frac{\gamma I_{m}(q)}{K_{m}\left(p^{1 / 2}\right)}-\frac{\Delta_{m} I_{m}\left(p^{1 / 2}\right)}{K_{m}\left(p^{1 / 2}\right)} \\
= & \gamma p^{1 / 2} I_{m} q I_{m}^{\prime}\left(p^{1 / 2}\right)-q I_{m}\left(p^{1 / 2}\right) I_{m}^{\prime}(q)
\end{aligned}
$$

Note that (A36) and (A37) make use of the relationship

The principle of orthogonality of cosine and sine functions can now be applied to evaluate the constants in (A30) and (A31). First, both sides of (A30) and (A31) are multiplied by $\sin m\left(\theta-\theta_{p w}\right)$ and integrated from $-\pi$ to $\pi$ to produce the following expressions for $m=0, \pm 1,2, \cdots$ :

$$
\begin{gathered}
C_{2 m} I_{m}(q)=C_{4 m} K_{m}\left(p^{1 / 2}\right) \\
q C_{2 m} I_{m}^{\prime}(q)=p^{1 / 2} \gamma C_{4 m} K_{m}^{\prime}\left(p^{1 / 2}\right)
\end{gathered}
$$

It can be shown through combination of (A32) and (A33) and use of recurrence relations for modified Bessel functions [Abramowitz and Stegun, 1972] that these equalities only hold if $C_{2 m}=C_{4 m}=0$. Both sides of (A30) and (A31) are then multiplied by $\cos m(\theta-\theta)$ and integrated from $-\pi$ to $\pi$ resulting in

$$
C_{1 m} I_{m}(q)=\frac{2}{p} I_{m}\left(p^{1 / 2}\right) K_{m}\left(p^{1 / 2} \xi_{p w}\right)+C_{3 m} K_{m}\left(p^{1 / 2}\right)
$$

$$
\begin{aligned}
q C_{1 m} I_{m}^{\prime}(q)=\frac{2 \gamma}{p^{1 / 2}} I_{m}^{\prime}\left(p^{1 / 2}\right) & K_{m}\left(p^{1 / 2} \xi_{p w}\right) \\
& +p^{1 / 2} \gamma C_{3 m} K_{m}^{\prime}\left(p^{1 / 2}\right)
\end{aligned}
$$

Since (A34) and (A35) consist of two equations with two unknowns, expressions for $C_{1 m}$ and $C_{3 m}$ can be readily found:
$I_{m}\left(p^{1 / 2}\right) K_{m}^{\prime}\left(p^{1 / 2}\right)-I_{m}^{\prime}\left(p^{1 / 2}\right) K_{m}\left(p^{1 / 2}\right)=-\frac{1}{(p)^{1 / 2}}$

which can be derived through combination of the Wronskian and recurrence relations for derivatives of modified Bessel functions [Abramowitz and Stegun, 1972].

These constants can now be substituted back into (A28) and (A29) resulting in the following expressions for $\bar{\phi}_{1}$ and $\overline{\phi_{d}}$ :

$$
\begin{aligned}
\overline{\phi_{1}}\left(\xi, \theta-\theta_{p w}, p\right)=\frac{2 \gamma}{p} & \sum_{-\infty}^{\infty} \frac{K_{n}\left(p^{1 / 2} \xi_{p w}\right)}{\Delta_{n}} \\
& \cdot I_{n}(q \xi) \cos n\left(\theta-\theta_{p w}\right)
\end{aligned}
$$

$$
\begin{gathered}
\overline{\phi_{d}}\left(\xi, \theta-\theta_{p u^{\prime},} p\right)=\frac{2}{p} \sum_{-\infty}^{\infty} \frac{\Theta_{n}}{\Delta_{n}} \\
\cdot K_{n}\left(p^{1 / 2} \xi_{p w^{\prime}}\right) K_{n}\left(p^{1 / 2} \xi\right) \cos n\left(\theta-\theta_{p w}\right)
\end{gathered}
$$

Substitution of (A40) and (A18) into (A19) produces the following expressions for $\overline{\phi_{2}}$ :

$$
\begin{aligned}
\overline{\phi_{2}} & =\frac{2}{p} \sum_{-\infty}^{x} \frac{K_{n}\left(p^{1 / 2} \xi_{p w}\right)}{\Delta_{n}}\left[I_{n}\left(p^{1 / 2} \xi\right) \Delta_{n}\right. \\
& \left.+\theta_{n} K_{n}\left(p^{1 / 2} \xi\right)\right] \cos n\left(\theta-\theta_{p w}\right), \quad \xi<\xi_{p w}
\end{aligned}
$$




$$
\begin{aligned}
\overline{\phi_{2}} & =\frac{2}{p} \sum_{-\infty}^{\infty} \frac{K_{n}\left(p^{1 / 2} \xi\right)}{\Delta_{n}}\left[I_{n}\left(p^{1 / 2} \xi_{p w}\right) \Delta_{n}\right. \\
& +\Theta_{n} K_{n}\left(p^{1 / 2} \xi_{p w}\right) \cos n\left(\theta-\theta_{p w}\right), \quad \xi>\xi_{p w}
\end{aligned}
$$

Note that the form of (A39) and (A41) allows ready comparison to the solution of Jaeger [1944], which, when written for a constant line source, is

$$
\begin{aligned}
& \overline{\phi_{1}^{*}}\left(\xi, \theta-\theta_{p w}, p\right)=\frac{2}{p} \sum_{-\infty}^{\infty} \frac{I_{n}\left(p^{1 / 2} \xi\right)}{\Delta_{n}^{*}}\left[K_{n}\left(p^{1 / 2} \xi_{p w}\right) \Delta_{n}^{*}\right. \\
& \left.-\Omega_{n}^{*} I_{n}\left(p^{1 / 2} \xi_{p w}\right)\right] \cos n\left(\theta-\theta_{p w}\right) \\
& \xi_{p w}<1, \quad 0 \leq \xi_{p w}<\xi_{p w} \\
& \overline{\phi_{1}^{*}}\left(\xi, \theta-\theta_{p w}, p\right)=\frac{2}{p} \sum_{-\infty}^{\infty} \frac{I_{n}\left(p^{1 / 2} \xi_{p w}\right)}{\Delta_{n}^{*}}\left[K_{n}\left(p^{1 / 2} \xi\right) \Delta_{n}^{*}\right. \\
& \left.-\Omega_{n}^{*} I_{n}\left(p^{1 / 2} \xi\right)\right] \cos n\left(\theta-\theta_{p w}\right), \\
& \xi_{p w}<\xi \leq 1
\end{aligned}
$$

$$
\begin{aligned}
& \overline{\phi_{2}^{*}}\left(\xi, \theta-\theta_{p w}, p\right)=\frac{2 \rho^{1 / 2}}{p} \sum_{-\infty}^{\infty} \frac{I_{n}\left(p^{1 / 2} \xi_{p w}\right)}{\Delta_{n}^{*}} \\
& \cdot K_{n}\left(\frac{p^{1 / 2}}{\rho^{1 / 2}} \xi\right) \cos n\left(\theta-\theta_{p w}\right), \quad \xi_{p w}<1,1 \leq \xi
\end{aligned}
$$

where

$$
\begin{gathered}
\overline{\phi_{i}^{*}}=\frac{4 \pi T_{1}}{Q} s_{i} \\
\Delta_{n}^{*}=q I_{n}^{\prime}\left(p^{1 / 2}\right) K_{n}\left(\frac{p^{1 / 2}}{\rho^{1 / 2}}\right)-p^{1 / 2} \gamma I_{n}\left(p^{1 / 2}\right) K_{n}^{\prime}\left(\frac{p^{1 / 2}}{\rho^{1 / 2}}\right) \\
\Omega_{n}^{*}=q K_{n}\left(\frac{p^{1 / 2}}{\rho^{1 / 2}}\right) K_{n}^{\prime}\left(p^{1 / 2}\right)-p^{1 / 2} \gamma K_{n}\left(p^{1 / 2}\right) K_{n}^{\prime}\left(\frac{p^{1 / 2}}{\rho^{1 / 2}}\right) .
\end{gathered}
$$

Note that in (A42) and (A43) the pumping well is located within the disk so the dimensionless drawdown is redefined using the transmissivity of the disk.

A quick check on the Laplace-space solution can be obtained by evaluating (A39) for the case of an observation well at the center of the disk $(\xi=0)$ :

$$
\begin{aligned}
\bar{\phi}_{1}\left(0, \theta-\theta_{p w}, p\right)=\frac{2 \gamma K_{0}\left(p^{1 / 2} \xi_{p w}\right)}{p \Delta_{0}} \\
=\frac{2 \gamma K_{0}\left(p^{1 / 2} \xi_{p w}\right)}{p\left[I_{1}(q) K_{0}\left(p^{1 / 2}\right)+\frac{p^{1 / 2} \gamma}{q} K_{1}\left(p^{1 / 2}\right) I_{0}(q)\right]} \frac{1}{q}
\end{aligned}
$$

The principle of generalized reciprocity [Bruggeman, 1972] holds that drawdown in the matrix for the case of a pumping well sited at the center of the disk should be equivalent to
(A44). Comparison of (A44) with (17) of Butler [1988] shows that such equivalence does hold. Note that (17) of Butler [1988] must be nondimensionalized and the numerator reduced by the Wronskian

$$
K_{0}(q) I_{1}(q)+K_{1}(q) I_{0}(q)=1 / q
$$

in order to get the same form as (A44). The Laplace-space solutions were also checked by substitution into (A10)(A17). The solutions honored the governing equations and boundary conditions in all cases.

The final step of the Laplace-transformation approach is to analytically invert the Laplace-space expressions back to real space. As is shown in Appendix B, this analytical back transformation is quite tedious for equations of the complexity of (A39) and (A41)-(A43). Therefore numerical approximations of the inversion are normally employed. Several approaches have been proposed [e.g., Stehfest, 1970; Talbot, 1979] for the numerical inversion of Laplace-space expressions. As stated earlier, the algorithm of Stehfest [1970] is employed here. Liu and Butler [1990] have written a series of Fortran programs that implement the numerical inversion scheme of Stehfest as well as the analytical inversion schemes of Appendix B. In Figure 2 and all the other cases examined for this work, the drawdown computed by the Stehfest algorithm closely matched the analytical drawdown except at very small times where the difference between the two approached one percent of the analytical drawdown. The efficiency of the two schemes was quite different, however, as the numerical integration needed for the analytical inversion required several minutes of CPU time on a Data General MV20000 minicomputer, while the Stehfest algorithm required less than a second. Note that 16 terms were used in the summation of the Stehfest algorithm for all the cases examined in this work.

\section{REFERENCES}

Abramowitz, M., and I. A. Stegun (Eds.), Handbook of Mathematical Functions, 1046 pp., Dover, New York, 1972.

Barker, J. A., and R. Herbert, Pumping tests in patchy aquifers, Ground Water, 20(2), 150-155, 1982.

Bear, J., Hydraulics of Groundwater, 567 pp., McGraw-Hill, New York, 1979.

Bruggemen, G. A., The reciprocity principle in flow through heterogeneous porous media, in Fundamentals of Transport Phenomena in Porous Media, pp. 136-149, Elsevier, New York, 1972.

Butler, J. J., Jr., Pumping tests in nonuniform aquifers-The radially symmetric case, $J$. Hydrol., $I 01(1 / 4), 15-30,1988$.

Butler, J. J., Jr., The role of pumping tests in site characterization: Some theoretical considerations, Ground Water, 28(3), 394-402, 1990.

Butler, J. J., Jr., A stochastic analysis of pumping tests in laterally nonuniform media, Water Resour. Res., 27(9), 2401-2414, 1991.

Butler, J. J., Jr., and W. Z. Liu, Pumping tests in non-uniform aquifers-The linear strip case, J. Hydrol., 128, 69-99, 1991.

Butler, J. J., Jr., and C. D. McElwee, Variable-rate pumping tests for nonuniform aquifers, Water Resour. Res., 26(2), 291-306, 1990.

Butler, J. J., Jr., W. Z. Liu, and C. D. McElwee, Flow to a well in radially nonuniform media, Open File Rep. 89-36, 31 pp., Kansas Geol. Surv., Lawrence, 1989.

Carslaw, H. S., and J. C. Jaeger, Conduction of Heat in Solids, 510 pp., Clarendon, Oxford, 1959.

Cooper, H. H., Jr., and C. E. Jacob, A generalized graphical method for evaluating formation constants and summarizing wellfield history, Eos Trans. AGU, 27(4), 526-534, 1946.

Doetsch, G., Guide to the Applications of Laplace Transforms, 255 pp., D. Van Nostrand, Princeton, N. J., 1961. 
Grader, A. S., and R. N. Horne, Interference testing: Detecting a circular impermeable or compressible subregion, SPE Form. Eval., 3(2), 420-428, 1988.

Gray, A., G. B. Mathews, and T. M. Macrobert, A Treatise on Bessel Functions and Their Applications to Physics, 327 pp., MacMillan, New York, 1952.

Haberman, R., Elementary Applied Partial Differential Equations, 547 pp., Prentice-Hall, Englewood Cliffs, N. J., 1987.

Hantush, M. S., Hydraulics of wells, in Advances in Hydrosciences, vol. 1, edited by V. T. Chow, pp. 281-432, Academic, San Diego, Calif., 1964.

Jaeger, J. C., Some problems involving line sources in conduction of heat, Philos. Mag. J. Sci., 35, 169-179, 1944.

Johnson, C. R., R. A. Greenkorn, and E. G. Woods, Pulse-testing: A new method for describing reservoir flow properties between wells, J. Pet. Technol., 18, 1599-1604, 1966.

Karasaki, K., Well test analysis in fractured media, Ph.D. dissertation, 239 pp., Univ. of Calif., Berkeley, 1986.

Larkin, B. K., Solutions to the diffusion equation for a region bounded by a circular discontinuity, Soc. Pet. Eng. J., 3(2), $113-115,1963$.

Liu, W. Z., and J. J. Butler, Jr., Software for the evaluation of analytical and semi-analytical solutions for pumping-induced drawdown in complex geologic settings, KGS Comput. Program Ser. 90-4, 52 pp., Kansas Geol. Surv., Lawrence, 1990.

McElwee, C. D., Sensitivity analysis of groundwater models, in Advances in Transport Phenomena in Porous Media, edited by $\mathrm{J}$. Bear and M. Y. Corapcioglu, NATO ASI Ser., Ser E, 128, 751-817, 1987.

McElwee, C. D., and M. A. Yukler, Sensitivity of groundwater models with respect to variations in transmissivity and storage, Water Resour. Res., 14(3), 451-459, 1978.

Moench, A., and A. Ogata, Analysis of constant discharge wells by numerical inversion of Laplace transform solutions, in Groundwater Hydraulics, Water Resour. Monogr., vol. 9, edited by J. Rosenshein, and G. D. Bennett, pp. 146-170, AGU, Washington, D. C., 1984 .

Stehfest, H., Numerical inversion of Laplace transforms, Commun. $A C M, 13(1), 47-49,1970$.

Streltsova, T. D., Well Testing in Heterogeneous Formations, 413 pp., John Wiley, New York, 1988.

Talbot, A., The accurate numerical inversion of Laplace transforms, J. Inst. Math. Appl., 23, 97-120, 1979.

Theis, C. V., The relation between the lowering of the piezometric surface and the rate and duration of discharge of a well using ground-water storage, Eos Trans. AGU, 16, 519-524, 1935.

Tongpenyai, Y., and R. Raghavan, The effect of wellbore storage and skin on interference test data, J. Pet. Technol., 33(1), 151-160, 1981.

Wheatcraft, S. W., and F. Winterberg, Steady state flow passing through a cylinder of permeability different from the surrounding medium, Water Resour. Res., 21(12), 1923-1929, 1985.

J. J. Butler, Jr. and W. Liu, Kansas Geological Survey, 1930 Constant Ave., Campus West, University of Kansas, KS 66047.

(Received March 4, 1992;

revised August 24, 1992;

accepted September 2, 1992.) 